\title{
Aspects Épidémiologiques, Diagnostiques Et Thérapeutiques Des Traumatismes Abdominaux À Bembéréké-Nord Bénin
}

\author{
Bio Tamou Sambo \\ Adrien Montcho Hodonou \\ Alexandre Salako Allode \\ Emile Mensah
}

Service de Chirurgie Générale, Centre Hospitalier Universitaire

Départemental du Borgou, Faculté de Médecine,

Université de Parakou (Bénin)

Mohamed Youssouf
Delphin Menhinto

Service de Chirurgie Viscérale, Centre National Hospitalier Universitaire de Cotonou, Faculté des Sciences de la Santé Universitéd'Abomey-calavi (Bénin)

\begin{abstract}
Objective: describe the epidemiological, diagnostic aspects and therapeutic approaches at Bembereke in northern Benin. Patients and methods: all victims of abdominal trauma received in the

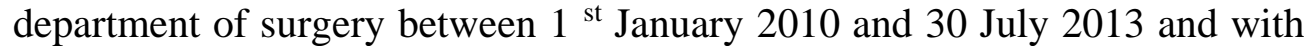
complete records were included in this retrospective study. Results: The abdominal trauma accounted for $1.1 \%$ of hospitalization and $10 \%$ of abdominal emergencies. The average age of patients was $28.04 \pm$ 22.25 years with extremes of 02 and 67 years. The sex ratio equaled to 7.17. Half of the patients were children under 15 years. The first three circumstances abdominal trauma was road traffic accidents 31 (31.63\%) cases, animal aggression $27(27.55 \%)$ cases and falls from a tree 14 $(14,29 \%)$ cases. Contusions were found in $73(74.50 \%)$ cases and wounds in $25(25.50 \%)$ cases. Fifty four $(55.10 \%)$ laparotomy were performed. The rate of white laparotomy is $5.55 \%$ with 3 cases. The spleen was the most affected organ (15\%) followed by small bowel (13\%). Morbidity was $8.16 \%$ dominated by parietal suppuration. The rate of mortality was $2.04 \%$. Mean hospital stay was 10.7 days.
\end{abstract}


Conclusion: abdominal trauma interested young adult male in northern Benin. Road traffic accidents and animal injuries were the leading causes.

Keywords: Trauma, abdominal, contusion, wound

\section{Résumé}

Objectif : Décrire les aspects épidémiologiques, diagnostiques, et thérapeutiques des traumatismes abdominaux à Bembéréké au nord du Bénin.

Patients et méthodes: Toutes les victimes de traumatisme abdominal reçues dans le service de chirurgie entre le $1^{\text {er }}$ Janvier 2010 et le 31 juillet 2013 et ayant un dossier complet étaient incluses dans cette étude rétrospective. Résultats: Les traumatismes abdominaux représentaient $1,1 \%$ des hospitalisés et $10 \%$ des urgences abdominales. L'âge moyen des patients était de 28,04 $\pm 22,25$ ans avec des extrêmes de 02 et 67 ans. La sex ratio était de 7,17. La moitié des traumatisés étaient des enfants de moins de 15 ans. Les trois premières circonstances de traumatisme abdominal étaient : les accidents de circulation $31(31,63 \%)$ cas, les agressions d'animaux 27 $(27,55 \%)$ cas et les chutes du haut d'un arbre $14(14,29 \%)$ cas. Il a été noté $73(74,50 \%)$ contusions et $25(25,50 \%)$ plaies. Cinquante quatre $(55,10 \%)$ laparotomies ont été réalisées. Le taux de laparotomie blanche était de $5,55 \%$ avec 3 cas. La rate était l'organe le plus touché (15\%) suivie du grêle (13\%). La morbidité était de 8,16\% dominée par les suppurations pariétales. Le taux de mortalité était de 2,04\%. La durée moyenne d'hospitalisation était de 10,7 jours.

Conclusion : les TA intéressent l'adulte jeune de sexe masculin au Nord Bénin. Les accidents de circulation et les traumatismes animaliers étaient les premières causes.

Mots clés: Traumatisme, abdominal, contusion, plaie

\section{Introduction}

Le traumatisme demeure la plus fréquente cause de mortalité dans les quatre premières décades de vie et est un problème de santé publique. L'abdomen est la troisième région la plus touchée nécessitant $25 \%$ des cas de chirurgie (Gad MA et al 2012). Les traumatismes abdominaux (TA) sont fréquents et potentiellement graves. Ils regroupent $80 \%$ des contusions et $20 \%$ des plaies en pratique civile (Gad MA et al 2012, Barth X et al 2001, Pailler JP 1991, Butt MU et al 2009). Ces traumatismes sont isolés dans 30 à $40 \%$ des cas (Barth X et al 2001), mais le plus souvent, ils s'accompagnent de lésions associées ou s'intègrent dans un contexte de poly traumatisme qui nécessite une approche multidisciplinaire. Quelles sont donc les spécificités 
épidémiologiques, diagnostiques et thérapeutiques des TA dans un contexte de pénurie du personnel qualifié, de plateau technique limité à l'hôpital de zone de Bembéréké dans le Nord Bénin.

\section{Cadre et Méthodes}

Cette étude a eu pour cadre le service de chirurgie générale de l'hôpital de Bembéréké. Cet hôpital confessionnel sans but lucratif fait office d'hôpital de zone desservant les communes de Bembéréké et de Sinendé, deux communes du nord Bénin peuplées de 213848 habitants (Institut National de la Statistique et de l'Analyse Economique RGPH 2013). Les activités champêtres et l'élevage sont les principales occupations des populations de ces communes. Le centre hospitalier de deuxième niveau de référence situé à Parakou est à cent dix kilomètres. Il s'agissait d'une étude rétrospective descriptive sur 03 ans et 07 mois allant $\mathrm{du} \quad 1^{\text {er }}$ janvier $2010 \quad$ au 31 Juillet 2013. Etaient inclus dans cette étude, tous les patients victimes d'un traumatisme abdominal (contusion ou plaie de l'abdomen) hospitalisés dans la période et ayant un dossier complet. N'étaient pas incluses, les victimes de TA qui n'étaient pas hospitalisées. Le diagnostic de plaie pénétrante était porté cliniquement devant une éviscération ou une épiplocèle, et confirmé après une laparotomie. Le diagnostic de contusion était évoqué devant une douleur ou éraflure abdominale avec notion de traumatisme à point d'impact abdominal, basi-thoracique ou pelvien. Les examens d'imagerie disponibles étaient la radiographie standard et l'échographie. Aucune ponction lavage du péritoine (PLP) n'a été faite. Les lésions viscérales étaient confirmées par les protocoles opératoires des patients opérés. Les paramètres suivant étaient analysés : sociodémographiques (âge, sexe, provenance), le mode d'admission, le délai de consultation, les étiologies, le traitement, l'évolution et la durée d'hospitalisation. Les données avaient été collectées à l'aide d'une fiche établie à cet effet. Les sources de collecte des données étaient les registres d'admission et du bloc opératoire puis les dossiers de malade. Les données recueillies étaient traitées avec les logiciels EPI DATA 3.1. Les logiciels EXCEL et WORD 2007 avaient servi à organiser des données sous forme de tableaux.

\section{Resultats}

Du $1^{\text {er }}$ janvier 2010 au 31 juillet 2013, soit 3 ans et 07 mois, 113 dossiers de TA ont été examinés dont 98 retenus. 


\section{Aspects épidémiologiques}

Durant cette période, 8898 malades avaient été hospitalisés dont 1021 pour des urgences chirurgicales abdominales. Avec 98 cas, les TA représentaient $1,1 \%$ des hospitalisations, $10 \%$ des urgences abdominales chirurgicales et en moyenne 27,4 cas par an.

L'âge moyen des patients était de 28,04 ans $\pm 22,25$ ans avec des extrêmes de 02 ans et 67 ans. Les enfants de moins de 15 ans étaient au nombre de 49 soit $50 \%$ de la population.

Il a été enregistré 86 hommes $(87,76 \%)$ et 12 femmes $(12,24 \%)$ soit une sex-ratio de 7,17.

Cinquante et quatre patients (55\%) provenaient des communes de la zone sanitaire : Bembéréké et Sinendé.

Soixante dix patients (71\%) étaient admis directement et $28(29 \%)$ sur référence.

Parmi ces patients, 74 (75,51\%) avaient consulté le même jour 21 $(21,3 \%)$ entre un et cinq jours et $3(3,06 \%)$ après cinq jours.

Les contusions abdominales étaient au nombre de 73 (74,50\%) et les plaies $25(25,50 \%)$ dont 11 étaient pénétrantes.

Les trois premières étiologies étaient respectivement : les accidents de circulation 31 cas $(31,63 \%)$, les traumatismes par les animaux en particulier les boeufs 27 cas $(27,55 \%)$ et les chutes du haut d'un arbre 14 cas $(14,29 \%)$. Le tableau I montre la répartition selon les étiologies des traumatismes de l'abdomen.

Tableau I : Répartition selon les étiologies des traumatismes de l'abdomen hospitalisés à

l'hôpital de zone de Bembéréké de janvier 2010 à juillet $2013(\mathrm{n}=98)$

\begin{tabular}{ccc}
\hline Variables & $\mathbf{N}$ & $\mathbf{\%}$ \\
\hline Accident de circulation & 31 & 31,63 \\
Traumatisme /Animal (bœuf) & 27 & 27,55 \\
Chute du haut d'un arbre & 14 & 14,29 \\
Rixes+braquage (agressions) & 12 & 12,25 \\
Accident de travail & 6 & 6,12 \\
Chute de sa hauteur & 3 & 3,06 \\
Jeu & 3 & 3,06 \\
Tentative d'autolyse & 2 & 2,04 \\
Total & $\mathbf{9 8}$ & $\mathbf{1 0 0}$
\end{tabular}

\section{Lésions associées}

Onze patients $(11,22 \%)$ avaient des lésions extra-abdominales. Neuf patients avaient des fractures. Les fractures intéressaient les membres chez 4 patients, le bassin chez 2 patients. Concernant les 3 autres cas de fractures, elles siégeaient sur une côte, une clavicule et la mandibule. L'hémothorax était retrouvé chez deux patients. Un cas de contusion abdominale était survenu sur une grossesse de six mois. 


\section{Aspects diagnostiques.}

Outre la clinique, les examens complémentaires à visé diagnostique étaient essentiellement la radiographie de l'abdomen sans préparation (ASP) réalisée pour 7 cas $(7,74 \%)$. L'échographie abdominale était faite pour 37 patients $(37,76 \%)$. La ponction trans-pariétale était réalisée sur 11 patients, soit $11,25 \%$.

\section{Aspects thérapeutiques}

Prise en charge médicale.

Tous les patients ont bénéficiés d'un abord veineux. Le remplissage vasculaire par des cristalloïdes et/ou les macromolécules était réalisé pour les patients à hémodynamique instable. La transfusion sanguine faite de sang total était indiquée pour les patients anémiés avec des signes de décompensation. Une dose de sérum antitétanique avait été administrée aux patients porteurs de plaie avec démarrage de la vaccination antitétanique. Les patients opérés avaient reçus une triple antibiothérapie faite d'ampicilline ou une céphalosporine de troisième génération associée à la métronidazole et la gentamicine. Tous les patients avaient bénéficiés d'antalgique.

Prise en charge chirurgicale

Toutes les plaies pénétrantes de l'abdomen avaient bénéficiées de laparotomie systématique.

Au total, 54 patients $(55,10 \%)$ avaient bénéficiés de laparotomie dont $03(5,56 \%)$ étaient blanches. Cinquante et un patients $(52,04 \%)$ avaient donc eu au moins une atteinte viscérale. La rate et le grêle étaient les plus touchés avec respectivement $18,36 \%$ et $15,30 \%$.

Le tableau II montre la répartition des lésions viscérales selon le type de traumatisme.

Tableau II : Répartition selon les viscères lésés et type de traumatisme abdominal des patients victimes de traumatismes abdominaux hospitalisés à l'hôpital de zone de Bembéréké.

\begin{tabular}{cccc}
\hline viscères lésés & Contusion & Plaie & Total \\
\hline Rate & 17 & 1 & 18 \\
Grêle & 9 & 6 & 15 \\
Colon + rectum & 4 & 1 & 5 \\
Foie & 4 & 0 & 4 \\
Mésentère & 5 & 0 & 5 \\
Vessie & 2 & 1 & 3 \\
Rein & 3 & 0 & 3 \\
Hématome retro péritoine & 7 & 0 & 7
\end{tabular}

\section{Association de lésions viscérales}

Neuf patients avaient au moins deux lésions viscérales associées : Un cas de plaie iléale associé une fissure de la rate et un cas de plaie colique 
associé à une contusion de la vessie. Tous les 07 cas d'hématome du rétro péritoine étaient associés a au moins une autre atteinte viscérale (foie, rate, vessie, iléon, mésentère).

Les gestes chirurgicaux réalisés étaient fonction des lésions constatées. Quinze cas de plaies iléales ont été enregistrés. Parmi ces cas, douze excisions sutures des berges des plaies avaient été réalisées contre trois résections suivies d'anastomose. La splénectomie totale était la règle en cas d'atteinte de la rate sauf pour un cas de fissure de la rate qui avait bénéficié de la pose d'un anti hémostatique type sugicel ${ }^{\circledR}$. Tous les hématomes retro péritonéaux (7) avaient été respectés. Le tableau III montre les actes de chirurgie réalisés lors des laparotomies.

Tableau III : Répartition des gestes chirurgicaux réalisés selon les atteintes viscérales des patients victimes de traumatismes abdominaux hospitalisés à l'hôpital de zone de Bembéréké.

\begin{tabular}{cccc}
\hline Lésions & $\mathrm{N}$ & Actes & $\mathrm{N}$ \\
\hline plaie du grêle & 15 & excision-suture & 12 \\
plaie colique & 3 & résection-anastomose & 3 \\
Contusion coliques & 2 & excision-suture & 3 \\
Rupture de rate & 17 & respect & 2 \\
Fissure de rate & 1 & Splénectomie totale & 17 \\
Plaie du foie & 4 & Pose de surgicèl & \\
& & «packing» & 1 \\
Plaie du mésentère & 1 & absence de geste & 2 \\
Hématome rétro péritonéal & 7 & Suture & 1 \\
\hline
\end{tabular}

Traitement des lésions extra-abdominales.

Deux patients avec hémothorax avaient bénéficiés de drain thoracique et un patient victime d'une fracture fermée de fémur avait bénéficié d'un enclouage centro-médullaire. Quatre patients avaient bénéficiés de traitement orthopédique pour fracture de membre et de la clavicule.

\section{Evolution}

Huit cas $(8,16 \%)$ de complications avaient été enregistrés dont 6 suppurations pariétales et 2 éviscérations post opératoires. Ces complications avaient évolué favorablement après traitement adapté. Deux patients 
$(2,04 \%) \quad$ victimes de poly traumatisme étaient décédés après deux jours d'hospitalisation..

\section{Durée d'hospitalisation}

La durée moyenne de séjour était de 10,7 jours avec des extrêmes de 1et 41 jours.

\section{Discussion}

\section{Aspects épidémiologiques}

\section{Fréquence}

Durant la période d'étude, les traumatismes abdominaux représentaient $1,1 \%$ des hospitalisés et $10 \%$ des urgences chirurgicales digestives dans le service. Harouna avait retrouvé un taux similaire de $9,89 \%$ des urgences chirurgicales digestives à Niamey (Harouna Y et al 2001). Par contre, Allode à Parakou avait trouvé que les TA représentaient 25,89\% des urgences chirurgicales (Allode SA et al 2011).

\section{L'âge}

L'âge moyen était de 28,04 $\pm 22,25$ ans avec des extrêmes de 02 ans et de 67 ans. La moitié (50\%) des traumatisés avaient un âge inférieur à 15 ans. Allode, à Parakou avait trouvé un âge moyen de 20,5 \pm 4 ans (Allode SA et al 2011). D'autres études avaient noté cette prédominance chez les sujets jeunes. Harouna avait retrouvé que $35 \%$ des TA à Niamey étaient des enfants de 5 à 15 (Harouna $Y$ et al 2001). Sani avait retrouvé que la proportion des enfants de 0 à 14 ans était de 41,1\% dans les contusions abdominales et 26,5 \% dans les plaies abdominales (Sani R 2004). La prédominance de cette tranche d'âge peut être liée à l'implication précoce des enfants dans les activités socioprofessionnelles (élevage, culture) en milieu rurale.

\section{Sexe}

Il a été noté une nette prédominance masculine $(87,7 \%)$ avec une sexratio de 7,17. Cette prédominance s'explique par l'activité socioprofessionnelle faisant que les hommes sont plus exposés que les femmes.

La prédominance masculine est conforme à la littérature : (Gad 2012, Harouna 2001, Allode 2011, Mnguni 2012, Groven 2012, Pekkari 2014, ElMenyar 2014)

\section{Aspects diagnostiques}

Etiologies: Les circonstances de TA décrites dans la littérature étaient retrouvées dans la série. Les accidents de circulation prédominent 
avec 33,63\% des étiologies (Gad 2012, Harouna 2001, Pekkari 2014, ElMenyar

Dans la série, la particularité du milieu rural (agro-pastoral) est la fréquence élevée de TA d'origine animale $(27,55 \%)$ et après chute du haut d'un arbre $(14,29 \%)$.

\section{Diagnostic lésionnel}

Les contusions représentaient $74,50 \%$ et les plaies, $25,50 \%$ des cas. La prédominance des contusions est rapportée dans plusieurs études dans les mêmes proportions (Gad 2012, Harouna 2001, Allode 2011, ElMenyar 2014, Hemmati 2013, Ayite 1996)

Par contre, Gaudeuille en Centrafrique et Mnguni à Durban en Afrique du Sud avaient trouvé respectivement $75 \%$ et $90,2 \%$ de plaies dues à la fréquence des agressions par arme blanche et à feu dans les grandes agglomérations (Gaudeuille 2007, Mnguni 2012).

Tous les cas d'éviscérations observés étaient survenus dans les traumatismes ouverts. Par contre, Bâ avait décrit un cas rare d'éviscération trans-anale du grêle due à une contusion abdominale par une brèche rectale (Bâ 2013).

\section{Les lésions viscérales}

Les organes pleins (rate, foie, reins) sont les plus atteints surtout dans les contusions. Ce constat est conforme à la littérature (Pailler 1991, Harouna 2001, Allode 2011, Sani 2004, Pekkari 2014, Menyar 2014)

Les organes creux (grêle, colon, vessie) étaient les seuls lésés en cas de plaies abdominales. D'autres études confirment la prédominence d'atteinte des viscères creux dans les plaies abdominales (Allode 2011, Madiba 2012, Gaudeuille 2007, Bâ 2013).

\section{Aspects thérapeutiques}

La stabilité hémodynamique, les résultats de l'examen clinique et de l'échographie ont permis un traitement non opératoire chez $33,67 \%$ des patients qui avaient une contusion abdominale. La laparotomie était systématique pour toutes les plaies pénétrantes de l'abdomen. La prise en charge des TA est en constante évolution. Le dogme de la laparotomie systématique dans les plaies de l'abdomen à évolué vers l'approche sélective non opératoire. Ces trente dernières années, l'affinage des procédures diagnostiques par l'étude de l'imagerie par résonance magnétique, la laparoscopie, le scanner et le FAST écho (focused abdominal sonography for trauma) ont contribué significativement pour la nouvelle tendance de la prise en charge des TA (Pailler 1991, Butt 2009). De même, 
la pratique de la radio intervention (embolisation de l'artère splénique) est d'un grand apport dans cette nouvelle politique.

La splénectomie totale était la règle (17/18) même si les indications décisionnelles de prise en charge se voient modifiés (Sani 2004, Dembele 2011, Ohene-Yeboah 2010).

Les lésions hépatiques avaient bénéficié d'un geste d'hémostase type "Packing» péri hépatique avec des compresses abdominales qui sont retirées après 48 à $96 \mathrm{H}$.

Les hématomes rétro péritonéaux avaient été respectés.

\section{Aspects évolutifs}

La durée moyenne d'hospitalisation a été de 10,7 jours avec des extrêmes de 1 et 41 jours. Cette durée était de 1 à 26 jours pour El-Menyal à Hamad au Quatar ( El-Menyar 2014).

Le taux de morbidité a été de $8 \%$ avec une prédominance de suppurations. Ce taux était de 6\% pour Mnguni à Durban en Afrique du sud (Mnguni 2012).

Le taux de mortalité était de $2 \%$. Un cas de décès a eu lieu à l'entrée, le diagnostic n'a pas été élucidé ; le second dans les premières 24 heures postopératoire chez un polytraumatisé. Le taux de mortalité était de $4,3 \%$ à Guilan en Iran (Hemmati 2013). Ce taux élevé s'explique par la gravité des lésions viscérales et l'association avec de traumatismes crâniens graves.

\section{Conclusion}

A Bembéréké, zone rurale du Nord du Bénin, les TA sont dominés par les contusions et intéressent le sujet jeune de sexe masculin. Les circonstances de survenues les plus fréquentes sont les accidents de circulation, les traumatismes engendrés par les bœufs et les chutes du haut d'un arbre. Une sensibilisation sur le respect du code de la route et à l'endroit des agriculteurs et éleveurs permettrait de réduire sensiblement le nombre de traumatisés abdominaux.

\section{References:}

Allode S.A., Olory-Togbé JL., Mensah E., et al 2011 Traumatismes abdominaux au C.H.D.U-P: Aspects Epidémiologiques et lésionnels Annales de l'université de Parakou Série : Sciences de la Santé 2 (2) 41-51 Ayite $\mathrm{A}$, Etey $\mathrm{K}$, Eteke L, et al Les plaies pénétrantes de l'abdomen à Lomé: à propos de 44 cas. Med Afr Noire. 1996;43(12):642-6. PubMed | Google Scholar

Barth X., LE Néel J-C., Guillon E. 2001 Traumatismes de l'abdomen. Rapport présenté au $103^{\text {ème }}$ congrès de chirurgie. Monographie de l'association française de chirurgie Paris Arnette 
Bâ PA, Diop B, Soumah SA, Aidara CM, Mbaye EM, Fall B. Les lésions intestinales au cours des traumatismes fermés de l'abdomen: diagnostic et prise en charge en milieu défavorisé. J Afr Hépatol Gastroentérol. 2013; 7(1):14-7. PubMed | Google Scholar

Butt M. U., Zacharias N., Velmaos G.C. 2009 Penetrating abdominal injuries: management controversies Scand J Trauma, Resusc Emerg Med , 17:19 PubMed | Google Scholar

Dembele B.T., Togo A., Diakité I., Kanté L., Traoré A., Cissé F., Coulibaly Y., KeïtaM., Diallo G. Perforations traumatiques d'organes creux intraabdominaux au CHU Gabriel-Touré. Journal Afr d'Hépato-Gastroentérologie, 2011; 5, (4) 290-292

El-Menyar A., Abdelrahman H., Al-Thani H.,et al Compartmental anatomical classification of traumatic abdominal injuries from academic point of view its potential clinical implication. Journal of Trauma Management \& Outcomes 2014, 8: 14 PubMed | PCM

Gad M.A., Saber A., Farrag S., Shams M.E., Ellabban G. M. 2012 Incidence, Pattern, and Factors Predicting Mortality of Abdominal Injuries in Trauma Patients N AMJ Med Sci 4(3): 129-134. PubMed | Google Scholar Gaudeuille A, Doui Doumgba A, Ndémanga Kamoune J, Sacko E, Nali NM. Abdominal trauma in Bangui (Central africa). Epidemiologic and et anatomical aspects. Mali Med. 2007; 22(2):19-22. PubMed | Google Scholar

Groven S., Gaarde C., Eken T., Skagan N. O., Naess P. A. Abdominal injuries in a major Scandinavian trauma center - performance assessment over an 8 year period Journal of Trauma Management \& Outcome 2014, 8:9 PubMed | Google Scholar

Harouna Y., Ali L., Seibou A., et al 2001 Deux ans de chirurgie digestive d'urgence à l'Hôpital National de Niamey (Niger) : Etude analytique et pronostique. Méd. Afr. Noire : 48 (2) 49-54

Hemmati H., Kazemnezhad-Leili E., Mohtasham-Amiri Z., et al 2013 Evaluation of Chest and Abdominal Injuries in Trauma Patients Hospitalized in the Surgery Ward of Teaching Hospital, Guilan, Iran Archives of Trauma Research 1 (4) 161-165 PubMed | Google Scholar

Institut National de la Statistique et de l'Analyse Economique : Résultats provisoires du recensement général de la population et de l'habitat 2013 (RGPH4) www.insae-bj.org

Leuge E., Wong L., Taylor J. 2007 Non-operative management for blunt splenic trauma in children: An updated literature review. Surgical Practice 11, 29-35

Mnguni M. N., Muckart D.J.J., Madiba T. E. 2012 Abdominal Trauma in Durban, South Africa: Factors Influencing Outcome Int Surg 97: 161-168 PubMed | Google Scholar 
Ohene-Yeboah M., Dakubo J. C. B., Boakye F. Naeeder S. B. 2010 Penetrating Abdominal Injuries in adults sen at twoo teaching hospitals in Ghana Ghana Medical Journal 44,3,103-108

Pailler J-L Traumatisme de l'abdomen in P.-L. Fagniez et D. Houssin, Pathologie chirurgicale Chirurgie Digestive et Thoracique (2) 48-53 Paris, Elsevier Masson 1991

Pekkari P., Bylund P-O. , Lindgren H., Oman M. Abdominal injuries in low trauma volume hospital - a descriptive study from northern Sweden Scandinavian Journal of Trauma and Emergency Medecine 2014, 22:48 PubMed | Google Scholar

Sani R., Ngo Bissemb NM, Bade MA, Baoua BM, Illo A, Bazira L. 2004 Les contusions de l'abdomen. Revue de 360 dossiers à l'Hôpital National de Niamey -Niger Méd. Afr. Noire 51 (10) 505-508

Sani R., Ngo Bissemb NM., IlloA., Souna B., Baoua BM, Bazira L. 2004 La plaie abdominale. Revue de 316 dossiers à l'Hôpital National de Niamey Niger. Méd. Afr. Noire 51 (7) 399-402 